\title{
Der Realität ins Auge schauen!
}

Die Schweiz verzeichnet fast 1500 Suizide pro Jahr, davon geschehen 30\% mit Hilfe einer Feuerwaffe, das ist mehr als ein Fall pro Tag. Ein enormes Ausmass. Weltweit an zweitoberster Stelle zu stehen, ist kein Ruhmesblatt ... und führt zu medizinischen und sozialen Auswirkungen, gegen die wir Ärztinnen und Ärzte unbedingt ankämpfen müssen.

Denn die Art und Weise der Selbsttötung ist nicht ohne Bedeutung, weder in ihren medizinischen Konsequenzen noch in ihren Auswirkungen auf die Anzahl der Suizide.

Aus Sicht der Medizin ist es eine Tatsache, dass Selbsttötungen mit Feuerwaffen schrecklich effizient sind. Und, wenn es nicht zum sofortigen Tod kommt, verursachen sie fürchterliche Schädigungen.

Bezüglich Gesundheitswesen kann es uns Ärztinnen und Ärzte in der Schweiz auch nicht gleichgültig sein, denn es ist bekannt, dass die Zahl der Suizide direkt mit der Anzahl Haushalte korreliert, in denen eine Feuerwaffe vorhanden ist.

Eine Waffe mehr oder weniger griffbereit zu Hause zu haben, kann natürlich in keiner Weise die Impulsivität einer Krisensituation zügeln, seien nun Alkohol oder Psychopharmaka mit im Spiel oder nicht. Die Möglichkeit des Zurückschreckens im letzten Moment oder der kurze Augenblick, der einem die Augen öffnet, kann also oft nicht stattfinden. Diese Überlegung - und das liegt der FMH ebenso am Herzen - gilt natürlich auch für die häusliche Gewalt.

Deshalb führt also ein hoher Anteil an vorhandenen Feuerwaffen in Haushalten zu einem Anstieg der Anzahl Suizide - eine traurige Logik, die von den verfügbaren Statistiken derjenigen Länder, die strengere Waffengesetze eingeführt haben, unbestreitbar belegt wird: Kanada, Australien, Grossbritannien ... in all diesen Ländern ist dank schärferer Gesetzgebung die Zahl der Suizide mit Feuerwaffen im gleichen Verhältnis zu den Haushalten, in denen Waffen vorhanden sind, zurückgegangen - um zwei Drittel!

Die Delegiertenversammlung der FMH hat sich an ihrer Sitzung vom 7. Juni 2007 mit dieser schwierigen Realität befasst und beschlossen, ihr ins Auge zu schauen.

Jetzt, wo eine Volksinitiative ergriffen wurde, die verlangt, dass der Waffenbesitz an einen Nachweis für die Notwendigkeit und die dafür erforderlichen Fähigkeiten geknüpft wird und Armeewaffen nur noch in gesicherten Räumen der Armee zu lagern sind, und nun, wo die Unterschriftensammlung angelaufen ist, will sich die FMH den Tatsachen nicht entziehen. Sie hat beschlossen, diese Initiative zu unterstützen.

Die Suizidprävention ist eine wichtige medizinische Aufgabe, nicht nur für Psychiater, und die in der Initiative vorgeschlagenen Massnahmen zeugen von gesundem Menschenverstand. Es gibt wirklich keinen Grund, nicht ein Waffenregister führen zu wollen, den Kauf einer Waffe nicht an einen Nachweis für die Notwendigkeit zu knüpfen und von denen, die eine Waffe tragen, nicht zu verlangen, dass sie den korrekten Umgang damit kennen; und es gibt heute auch keinen Grund mehr zu glauben, die Armee sei schlagkräftiger, wenn die Gewehre zu Hause aufbewahrt werden statt in einem Zeughaus.

Die Delegiertenversammlung der FMH hat entschieden, der Realität ins Auge zu schauen und die Initiative zu unterstützen - eine gute Entscheidung, die von gesundem Menschenverstand zeugt.

Dr. med. Jacques de Haller, Präsident der FMH 